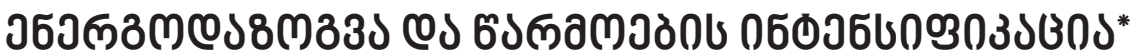

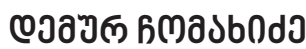

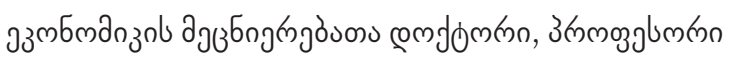

https://doi.org/10.35945/gb.2018.06.027

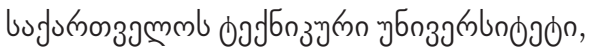

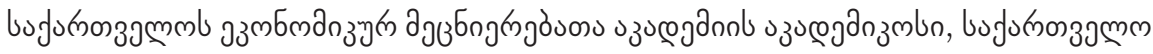

demurchomakhidze@yahoo.com

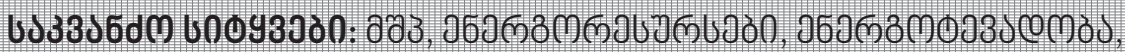

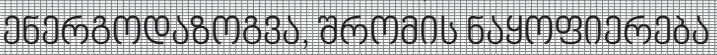

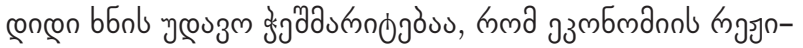

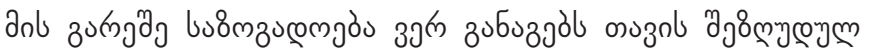

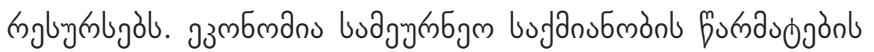

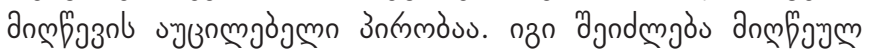

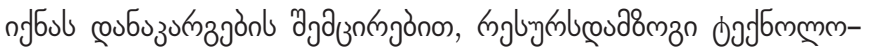

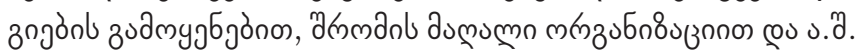

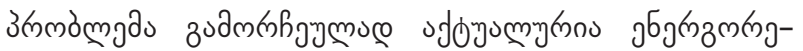

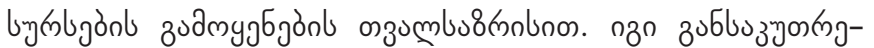

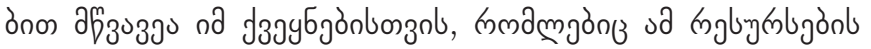

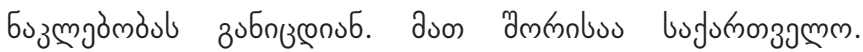

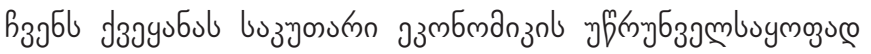

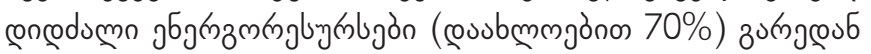

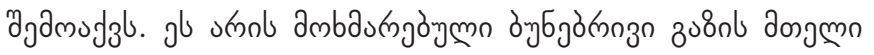

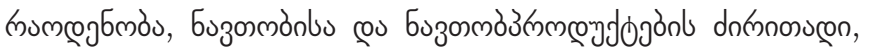

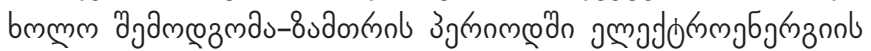

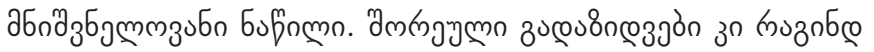

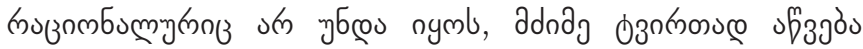

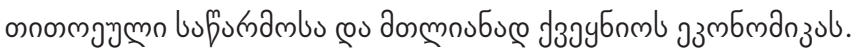

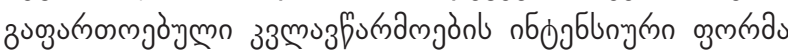

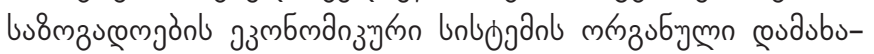

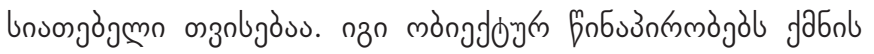

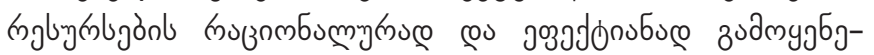

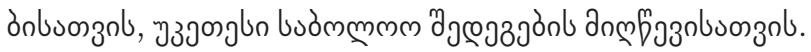

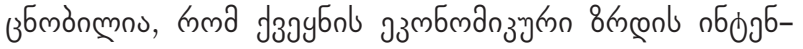

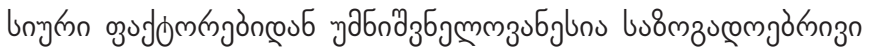

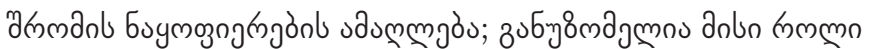

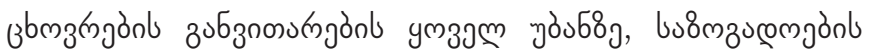

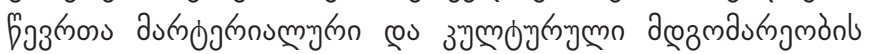

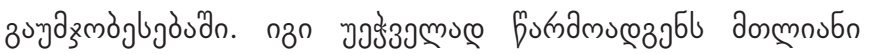

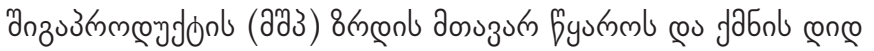

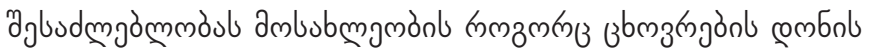

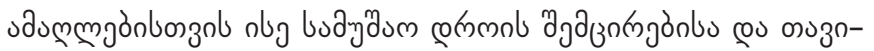

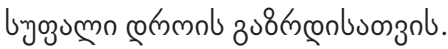

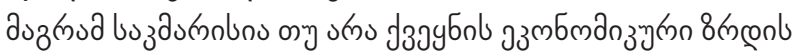

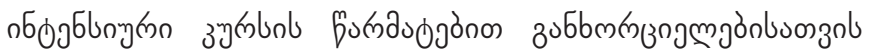

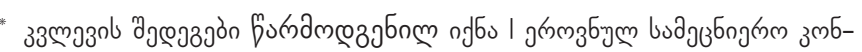

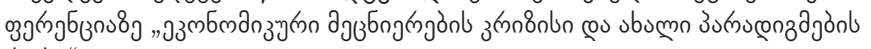
dngos"

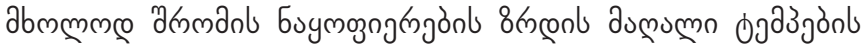

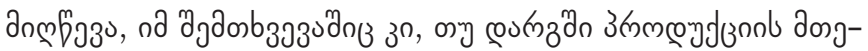

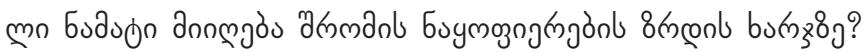

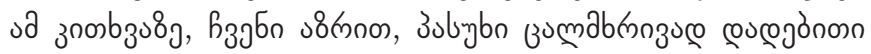

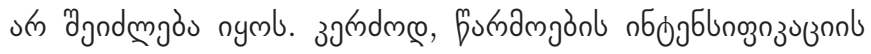

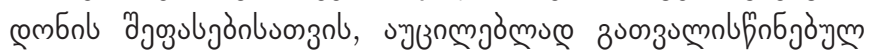

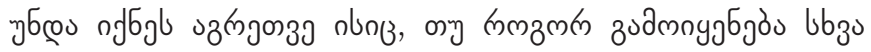
nglughbodo.

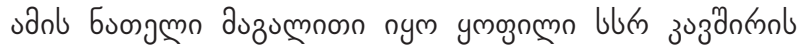

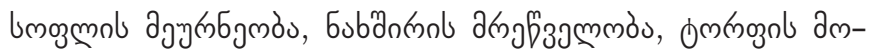

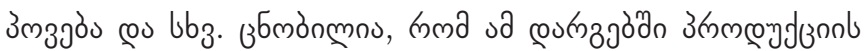

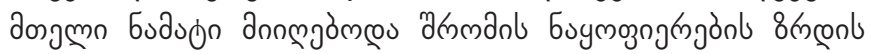

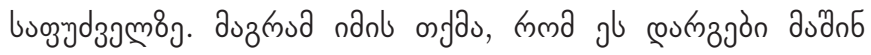

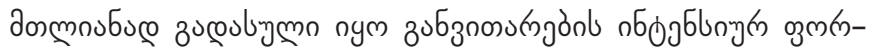

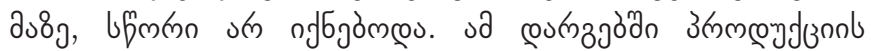

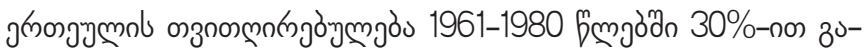

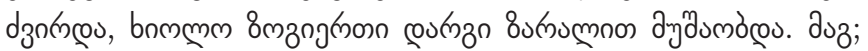

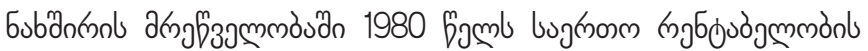

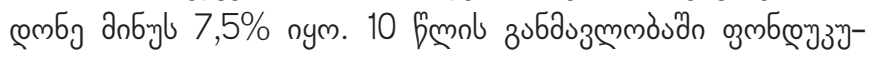

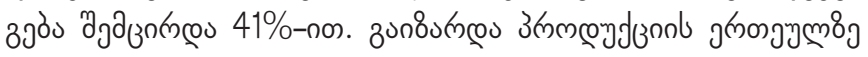

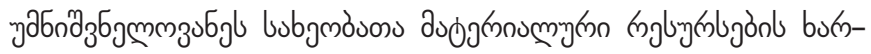

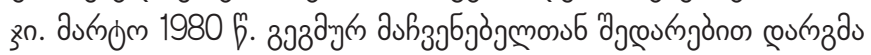

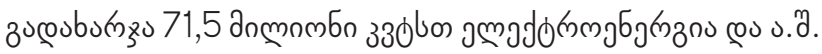

8дамm uмб

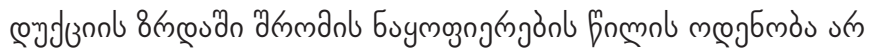

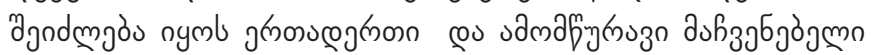

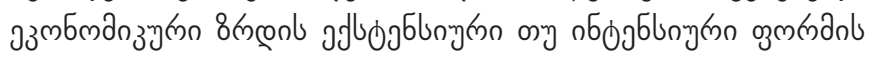

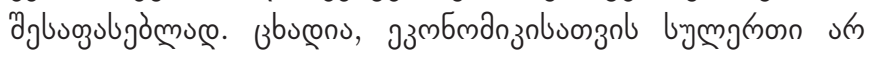

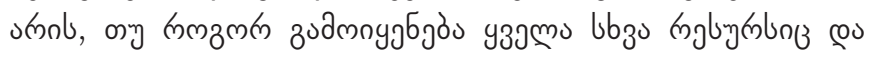

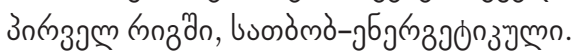

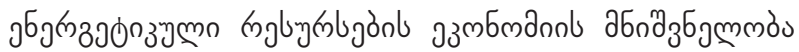

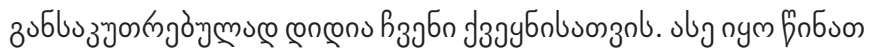

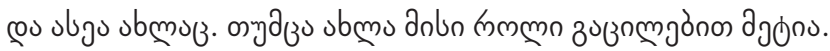

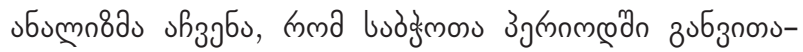

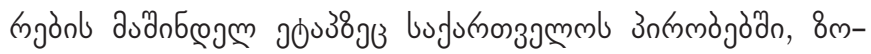

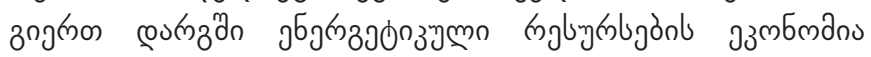

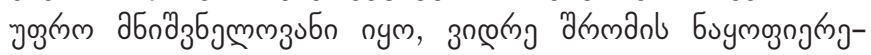
onl sduмm 


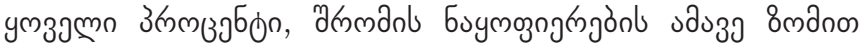

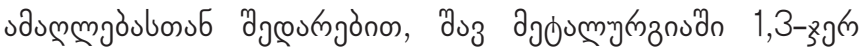

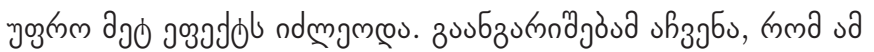

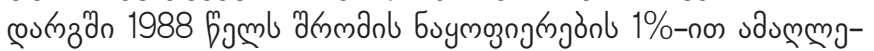

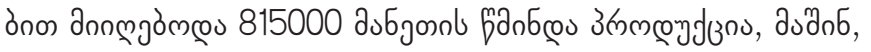

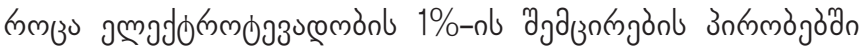

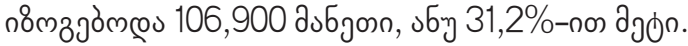

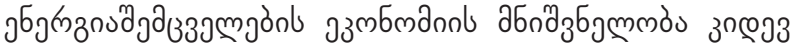

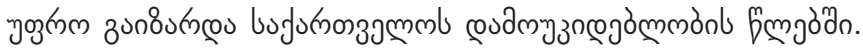

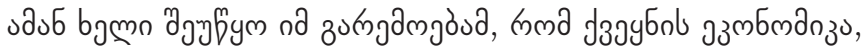

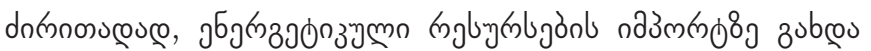

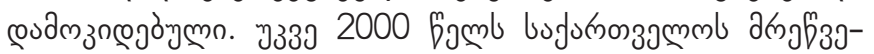

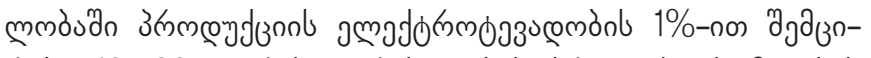

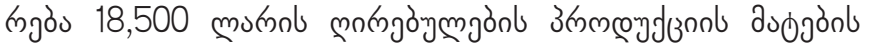

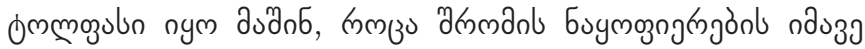

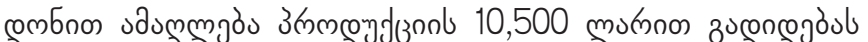

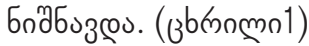

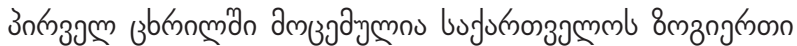

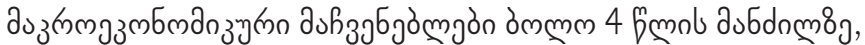

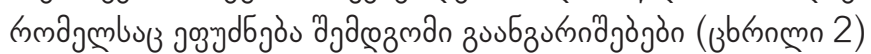

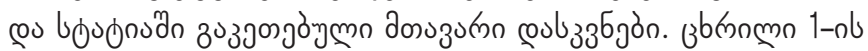

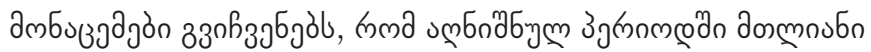

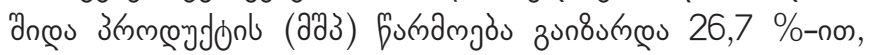

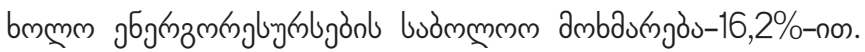

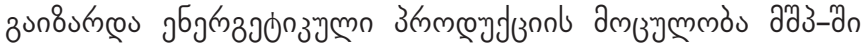

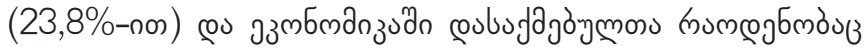

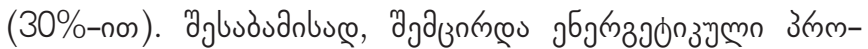

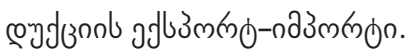

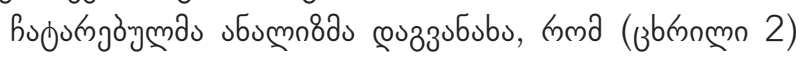

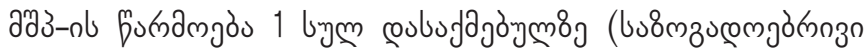

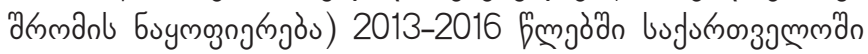

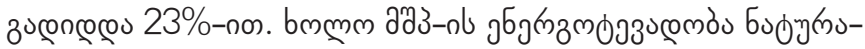

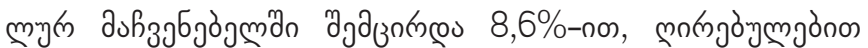
дง

उbronmo 1.

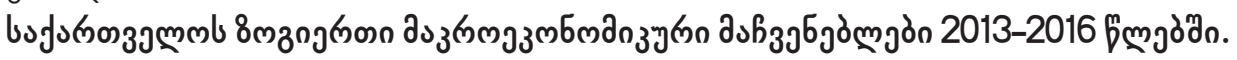

\begin{tabular}{|c|c|c|c|c|c|c|}
\hline \multirow{2}{*}{ 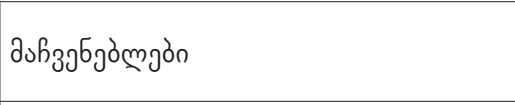 } & \multirow{2}{*}{ 8мдnl g๓олэмп } & \multicolumn{4}{|l|}{ fmagòn } & \multirow{2}{*}{$\begin{array}{l}\text { 2016f.\%-non } \\
\text { 2013f.--0ை6 }\end{array}$} \\
\hline & & 2013 & 2014 & 2015 & 2016 & \\
\hline 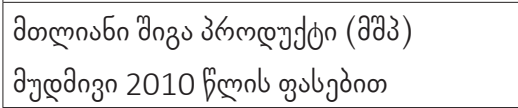 & dmb. mumo ${ }^{1}$ & 26847,4 & 29150,5 & 31755,6 & 34028,5 & 126,7 \\
\hline 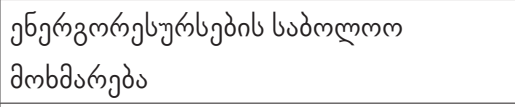 & 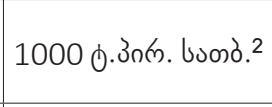 & 3726,3 & 4022,8 & 4174,6 & 4330,5 & 116,2 \\
\hline 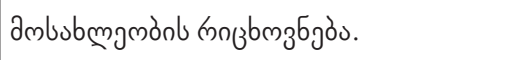 & 10003030 & 4483,8 & 4490,5 & 3713,2 & 3720,4 & 83,0 \\
\hline 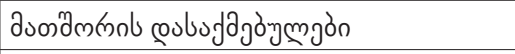 & 10003030 & 1712,1 & 1745,2 & 1779,9 & 1763,9 & 103,0 \\
\hline 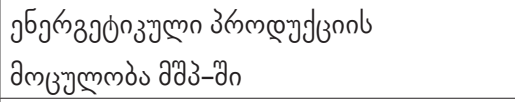 & amb. motno & 1075,6 & 1158,5 & 1147,0 & 1332,0 & 123,8 \\
\hline jб & 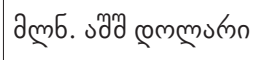 & 1291,5 & 1127,1 & 1172,9 & 975,4 & 75,5 \\
\hline 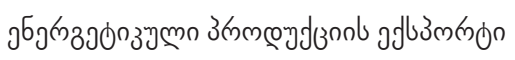 & 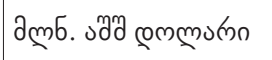 & 65,9 & 3,1 & 129,8 & 62,3 & 94,5 \\
\hline
\end{tabular}

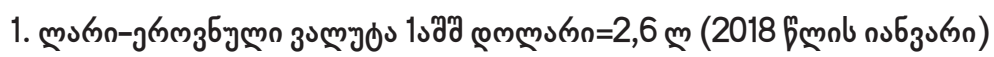

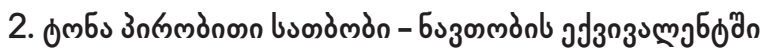

उbmanno 2.

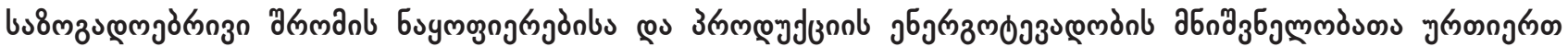

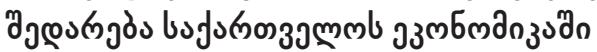

\begin{tabular}{|c|c|c|c|c|c|c|}
\hline \multirow{2}{*}{ дง } & \multirow{2}{*}{ 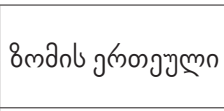 } & \multicolumn{4}{|l|}{ Cemgòn } & \multirow{2}{*}{ 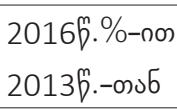 } \\
\hline & & 2013 & 2014 & 2015 & 2016 & \\
\hline 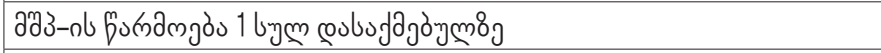 & mukno & 15681 & 16703 & 17841 & 19292 & 123,0 \\
\hline 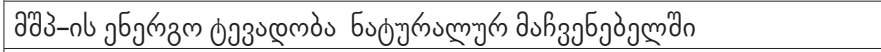 & $38.3 .6 /$ momo & 0,139 & 0,138 & 0,131 & 0,127 & 91,4 \\
\hline 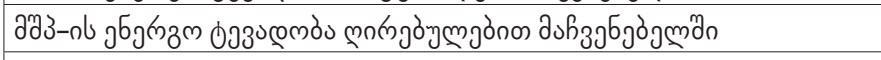 & motron & 0,118 & 0,109 & 0,108 & 0,106 & 89,8 \\
\hline 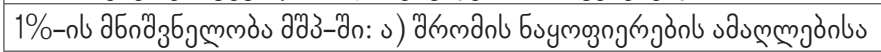 & dmb. mutmo & 267 & 290 & 256 & 429 & 160,6 \\
\hline 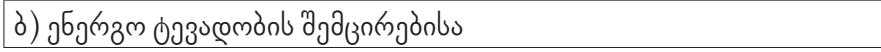 & amb.mukno & 208 & 313 & 301 & 400 & 192,3 \\
\hline
\end{tabular}




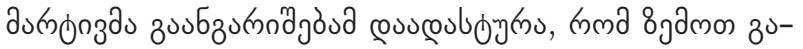

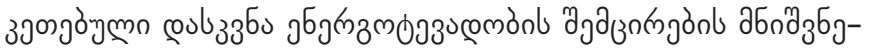

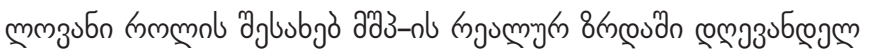

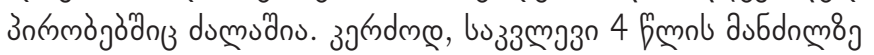

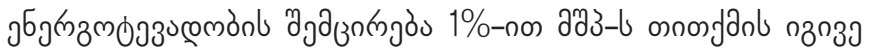

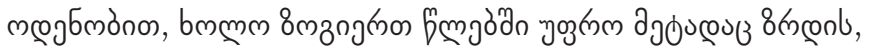

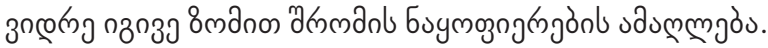

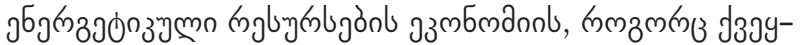

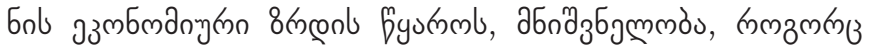

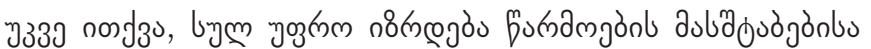
eu don n nб

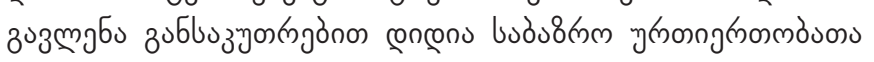

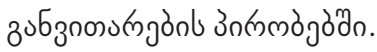

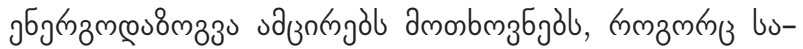

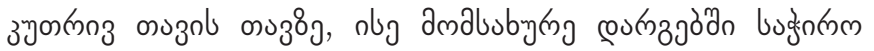

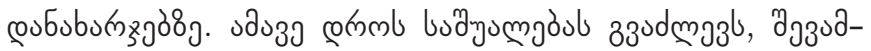

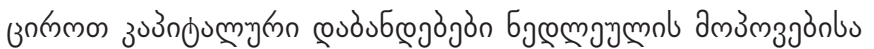

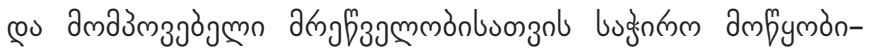

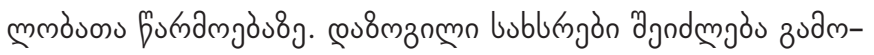

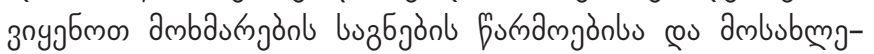

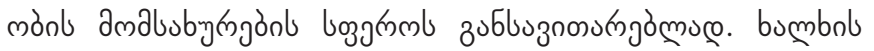

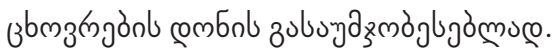

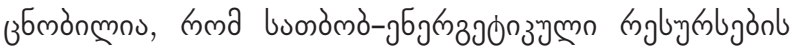

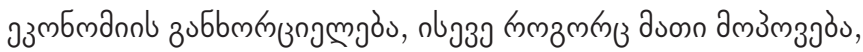

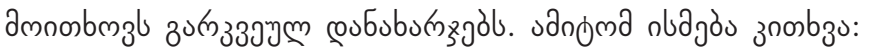

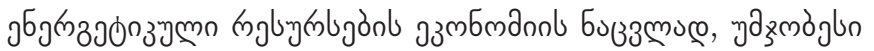

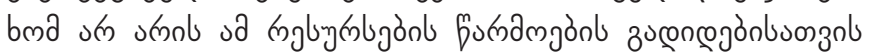
8 काच

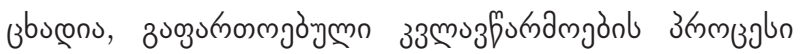

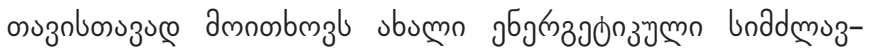

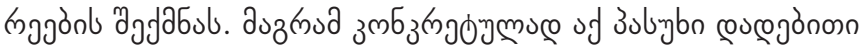

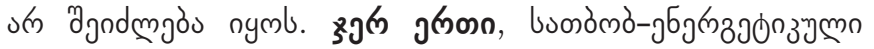

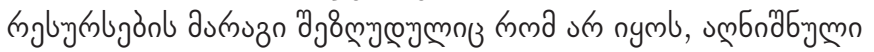

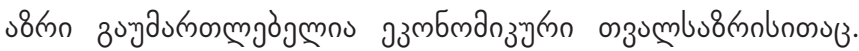

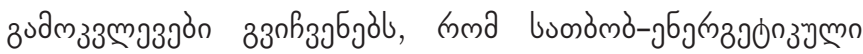

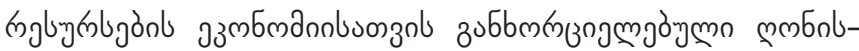

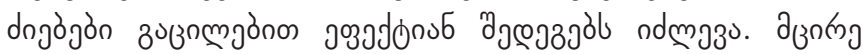

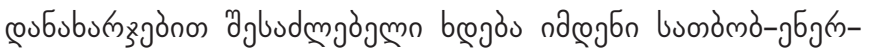

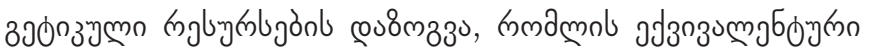

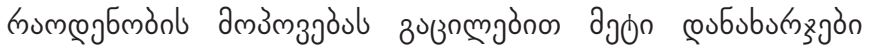

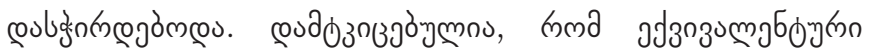

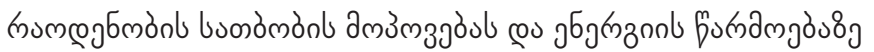

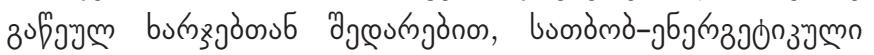
(n)

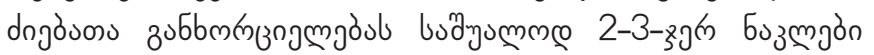
nsbbu lignnnegoso.

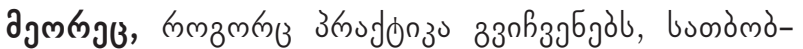

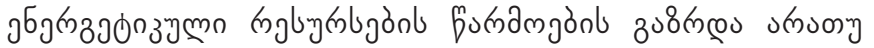
saznn

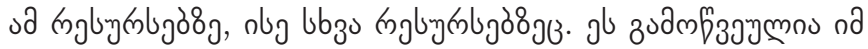

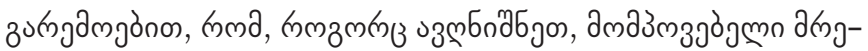

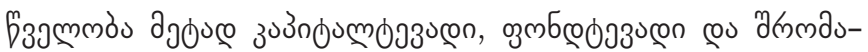

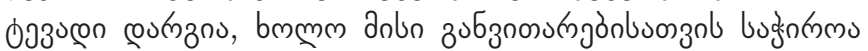
aбnazб

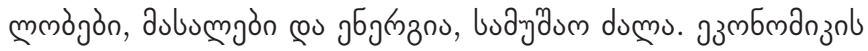

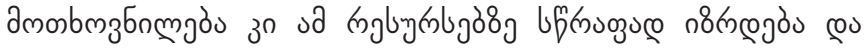
anbn eszaugmoुnm

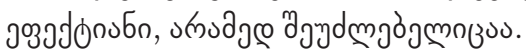

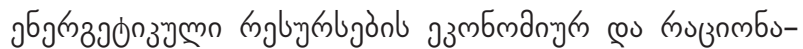

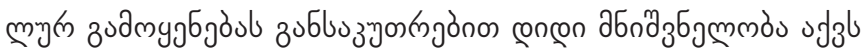

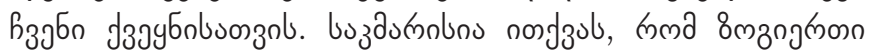

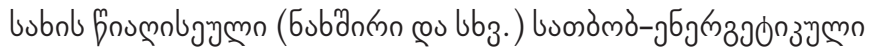

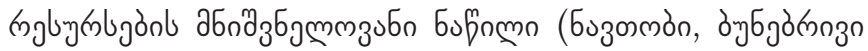

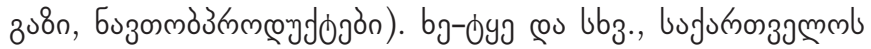

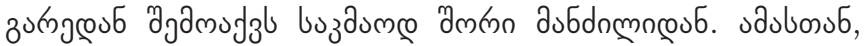

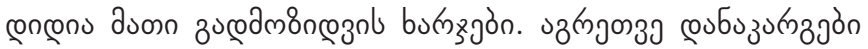

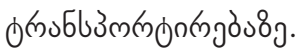

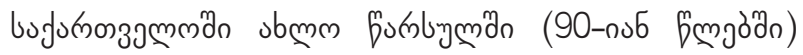

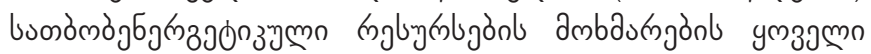

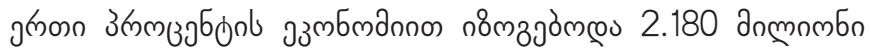

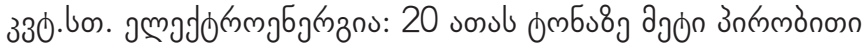

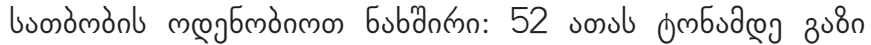

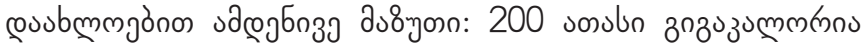

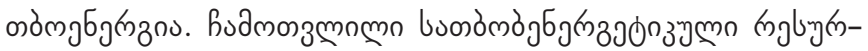

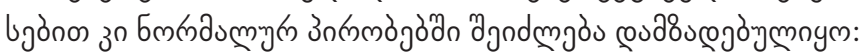

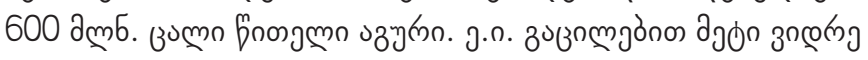

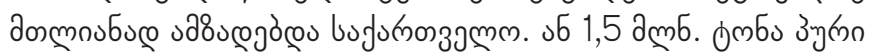

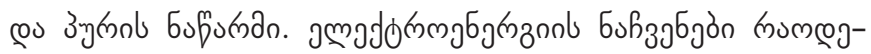

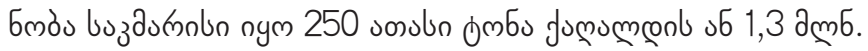

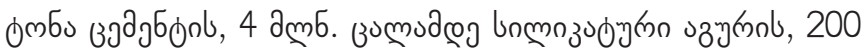

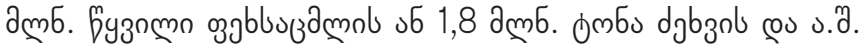
fornamgànbonzll.

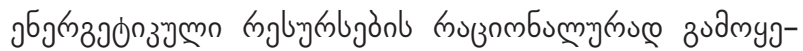

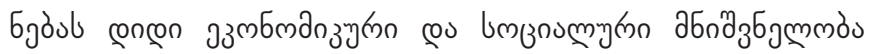

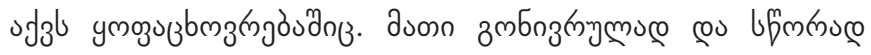

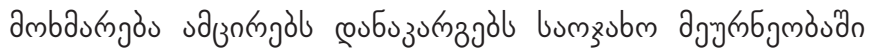

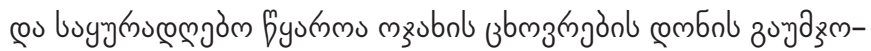

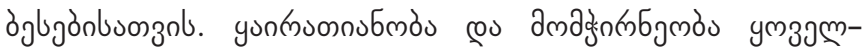

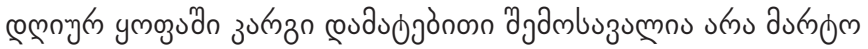

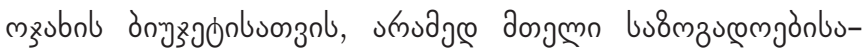

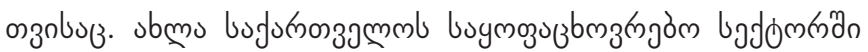

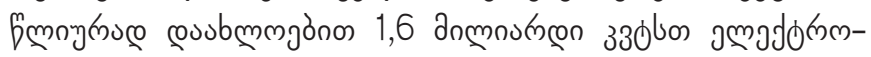

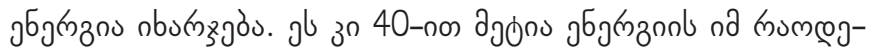

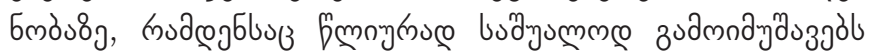

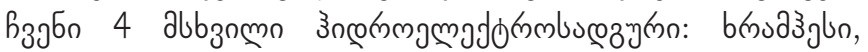

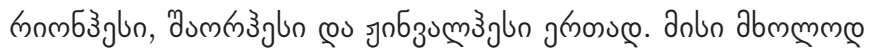

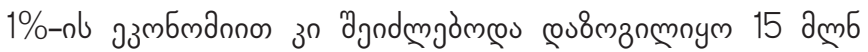

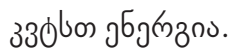




\section{ENERGY SAVING AND INTENSIFICATION OF PRODUCTION}

\section{DEMUR CHOMAKHIDZE}

https://doi.org/10.35945/gb.2018.06.027

Doctor of Economic Sciences, Professor

Georgian Technical University

Academician of Academy of Economic Sciences of Georgia, Georgia

demurchomakhidze@yahoo.com

KEYWORDS: GDP, ENERGY RESOURCES, ENERGY EFFICIENCY, ENERGY SAVING, LABOR PRODUCTIVITY

\section{SUMMARY}

The report analyzes the role and importance of energy conservation for Georgia. Based on concrete materials, the effectiveness of social labor and energy savings on gross domestic product (GDP) is comparable to each other. It is said that the reduction of GDP energy efficiency by $1 \%$ is almost the same, and in more than a few years, the result of GDP increase is more than the same as the productivity of public labor. In this regard, the report describes the level and dynamics of major macroeconomic indicators of the economic development of Georgia for 2013-2016. Set up events to improve the situation. 